\title{
SAT-ESPEC : Análise e Coleta de Dados da Transmissão de Estações Terrenas de uma Rede Satélite
}

\author{
Josinaldo Azevedo ${ }^{1}$, André L. C. Barcellos ${ }^{1}$, Amanda C. A. Mendes ${ }^{1}$, \\ Daniel de Oliveira ${ }^{2}$, Paulo C. S. Vidal ${ }^{1}$, Marcos Bedo ${ }^{3}$ \\ ${ }^{1}$ Instituto Militar de Engenharia (IME) \\ Rio de Janeiro - RJ - Brasil \\ ${ }^{2}$ Universidade Federal Fluminense (UFF) \\ Instituto de Computação - Niterói - RJ - Brasil \\ ${ }^{3}$ Universidade Federal Fluminense (UFF) \\ Instituto do Noroeste Fluminense - S. A. Pádua - RJ - Brasil \\ \{josinaldo, andrebarcellos, amendes, vidal\}@ime.eb.br \\ \{danielcmo@ic, marcosbedo@id\}.uff.br
}

\begin{abstract}
Satellite networks are an essential component of Brazilian telecommunications infrastructure. In particular, satellites are the only network connection between several Brazilian remote locations and outposts. This study provides a sampled dataset, coined SAT-ESPEC, of spectrograms from five Brazilian stations. It includes real spectrograms collected from earth stations placed in the North of South America. Data were collected from an operating satellite by a Radiomonitoring and Geolocation Station from the Brazilian National Telecommunications Agency (ANATEL) at Rio de Janeiro city. The dataset fits the domains of radio propagation as well as network security and can be used for tasks such as classification, outlier detection, and time-series analyses.

Resumo. As redes de satélites são um complemento essencial para a infraestrutura de telecomunicações no Brasil. Em alguns casos, esta rede é o único meio de comunicação para várias localidades remotas, que são de difícil acesso geográfico. Esse trabalho tem o objetivo de disponibilizar uma base, denominado SA T-ESPEC, construída a partir do espectrograma gerado da recepção dos sinais de cinco estações terrenas, que estão instaladas e em operação na Região Norte do Brasil. Toda a coleta de dados ocorreu numa rede em produção através da Estação de Radiomonitoração e Geolocalização da Agência Nacional de Telecomunicações (ANATEL), que fica no município do Rio de Janeiro. Esse conjunto se encaixa no domínio de propagação de radiofrequência e segurança de rede, e pode ser usado para tarefas como classificação de dados, detecção de anormalidades e análises de séries-temporais.
\end{abstract}

\section{Introdução}

O Brasil, por possuir dimensões continentais, apresenta desafios de conexão que outros países com extensão territorial menor não enfrentam. Um dos problemas que se apresentam é levar conectividade e redes de telecomunicações às regiões remotas e muito afastadas de centros urbanos. Em diversos casos, a única alternativa para superar esse enorme 
desafio logístico, para conectar milhares de brasileiros que vivem nessas localidades, é por meio do uso extensivo de redes por satélite.

Uma das aplicações mais conhecidas pela população em geral é o serviço de TV por assinatura via satélite (direct to home - DTH). Outro serviço, também bastante utilizado, é a recepção de canais de TV abertos por satélite (television receive-only - TVRO). Não obstante, satélites possuem diversas outras atribuições, como sua utilização para análise meteorológica, monitoramento e mapeamento de florestas, vigilância e segurança, e para levar serviços de telecomunicações e streaming, como celular e internet, em áreas que não podem ser atendidas por fibra óptica ou rádio enlace terrestre.

Consequentemente, o desenvolvimento e inclusão social e humana nessas regiões do país depende diretamente do uso dessas redes. Em concreto, as redes de satélite cobrem uma vastíssima área do país e da América do Sul com o papel de integrar as comunicações de regiões rurais e agrícolas, regiões amazônica, interior do Nordeste e pantanal, bem como ilhas e postos afastados na costa brasileira. Portanto, é de suma importância proteger e aperfeiçoar constantemente essa rede de comunicação, o que inclui desafios como evitar interferências, sinais "piratas" e outros usos indevidos da infra-estrutura.

Nesse sentido, a caracterização da ocupação e a identificação das tecnologias operacionais em uma banda de frequência definem a probabilidade de uma comunicação bem ou mal sucedida. Além disso, a identificação de tecnologias que utilizam canais específicos auxilia na modelagem de interferências encontradas ao tentar acessar o meio [Bitar et al. 2017]. Esse estudo disponibiliza uma base de dados inédita para fins de pesquisa, onde os sinais de cinco estações terrenas foram coletados através da utilização de espectrogramas. Essas estações estão instaladas em três estados da Região Norte do Brasil, a saber: Acre, Amazonas e Pará; e têm a finalidade de prover conectividade entre 05 (cinco) diferentes cidades. A identificação das cidades e dos satélites são ocultadas nesse trabalho por questões de legislação e privacidade. A base de dados, denominada SAT-ESPEC foi curada a partir de espectrograma coletados pela Estação de Monitoração de Satélites (EMSAT) da Agência Nacional de Telecomunicações (ANATEL), cuja instalação fica localizada na cidade do Rio de Janeiro/RJ - Brasil.

Os dados coletados cobrem a frequência de onda de sinais recebidos da rede de satélites brasileiros. Espectrogramas são usados em diversas tarefas computacionais, como na detecção de interferência na faixa de rádio frequência [Bitar et al. 2017], ou na identificação de padrões em eletrocardiogramas e eletroencefalogramas [Wang et al. 2019]. Também é comum encontrar tarefas associadas à classificação e agrupamento de espectrograma, tal como os estudos para reconhecimento de emoções contidas na fala ou em áudio [Özseven 2018]. Nesse sentido, argumenta-se que o conjunto de dados SAT-ESPEC pode ser usado em diferentes tarefas computacionais, incluindo classificação, detecção de anormalidades e análises de séries temporais. Ao final desse trabalho, um exemplo de classificação de sinal é apresentado para mostrar o potencial do conjunto de dados no treinamento de modelos de aprendizado.

O restante desse estudo está organizado da forma como se segue. A Seção 1 introduz as áreas de pesquisa relacionadas ao conjunto de dados. A Seção 2 aborda os conceitos básicos para explicar a construção da proposta e a coleta dos dados, enquanto a Seção 3 apresenta os dados coletados que compõem o conjunto SAT-ESPEC, bem como 
o tratamento que foi realizado para curar a base bruta. A Seção 4 traz algumas sugestões para a utilização do conjunto SAT-ESPEC , resultados preliminares de classificação do sinal e as considerações finais deste trabalho, enquanto a última Seção mostra as referências bibliográficas.

\section{Sinais Analisados: Origem e Características da Radiofrequência}

A seguir são apresentados a topologia da rede satélite - local da coleta dos dados - e as características de radiofrequência (RF) dos sinais recebidos e armazenados pela Estação de Monitoramento de Satélites Geoestacionários (EMSAT).

Topologia da Rede Satélite. A topologia de uma rede satélite possui três elementos: (1) a remota satélite, (2) o satélite e (3) a estação principal (ou HUB). A Figura 1 ilustra de forma simplificada esta topologia. Tanto a remota quanto o HUB também podem ser chamados de "estações terrenas" pela sua localização física.

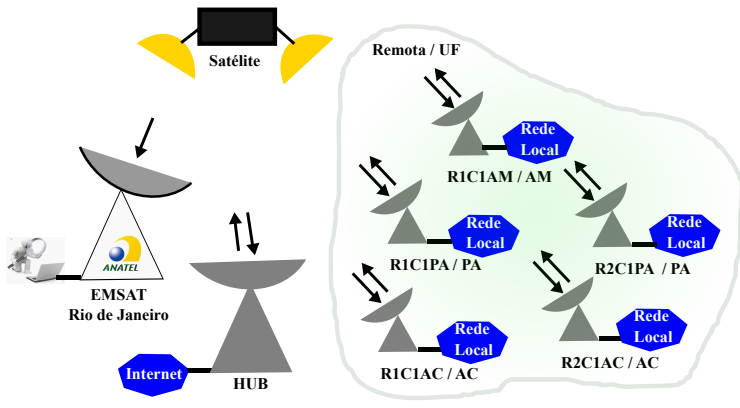

Figura 1. Topologia simplificada de uma Rede Satélite.

A Remota Satélite. A remota satélite é um conjunto de equipamentos que tem o objetivo de prover infraestrutura de telecomunicações num determinado local (e.g., ilhas, florestas, etc.), que usualmente não possui outro meio de conectividade. Esses equipamentos incluem: (1) modulador (onde é conectado o roteador da rede, por exemplo), (2) cabos de RF (radiofrequência), (3) BUC (block up converter) - transmissão, (4) LNB (low noise block) - recepção, e (5) antena - transmissão e recepção.

As remotas que tiveram seus sinais coletados neste trabalho são do tipo SCPC (single channel per carrier). Foram selecionadas cinco remotas satélites instaladas e operando no Brasil, sendo duas no Pará (uma estação com duas e outra com três portadoras ${ }^{1} /$ sinais transmitidos), duas no Acre (cada uma com duas portadoras transmitidas) e uma no Amazonas (com três portadoras). No total, foram 12 portadoras/sinais que tiveram seus dados coletados para a construção do conjunto SAT-ESPEC - Ver Tabela 1.

O Satélite. Um satélite é um objeto dividido em repetidores de radiofrequência, que por sua vez é simplesmente um receptor ligado a um transmissor, que usa diferentes frequências de rádio para transmitir e receber, que pode receber um sinal de uma remota satélite, amplificá-lo e retransmiti-lo para outra estação [Pratt and Allnutt 2020]. O satélite da rede analisado é Geoestacionário, segue uma trajetória circular em volta da terra, se localizando sobre a linha do Equador, numa distância de cerca de $36.000 \mathrm{Km}$.

\footnotetext{
${ }^{1}$ Sinal multidimensional de alta frequência onde são transportadas as informações do usuário.
} 
A HUB. É a estação principal da topologia de rede satélite, e normalmente está instalada em grandes centros urbanos, onde existe interligação com a Internet. As remotas se conectam a ela, numa topologia estrela, o que possibilita o acesso à web e demais serviços de redes orientadas a circuitos ou a pacotes.

Estação de Monitoramento de Satélites Geoestacionários. A estação de radiomonitoração e geolocalização da ANATEL (EMSAT) foi concebida no contexto da necessidade específica da indústria de satélite no Brasil - Portaria ANATEL n 1637 , de 23 de novembro de 2017. A radiomonitoração de satélites possui diversas particularidades frente às técnicas tradicionais de monitoração do espectro de serviços terrestres de radiocomunicação e, portanto, requer procedimentos específicos. Esta estação forneceu os recursos e instrumentos necessários para a coleta de dados dos sinais transmitidos das estações que foram selecionadas para esta pesquisa. A EMSAT encontra-se instalada na região da Ilha do Governador, cidade do Rio de Janeiro/RJ - Brasil (Figura 2(a)). Sua localização proporciona monitorar a maior parte dos satélites de comunicação geoestacionários autorizados a operar no Brasil.

A leitura dos sinais foi realizada em uma antena de banda $\mathrm{C}$, faixa de frequência de recepção 3,625 GHz a 4,2 GHz, que possui $6 \mathrm{~m}$ de diâmetro. Os sinais foram convertidos para a banda L (faixa de $950 \mathrm{MHz}$ a $1,525 \mathrm{GHz}$ ) na cadeia de RF da estação, coletados e armazenados no analisador de espectro de alto desempenho de tempo real FSVR da Rohde \& Schwarz (R\&S). A Figura 2 (b) apresenta a imagem do analisador de espectro utilizado na coleta da base de dados.

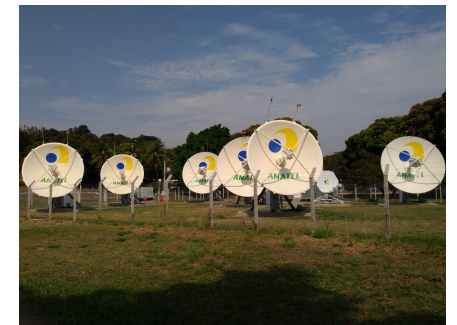

(a)

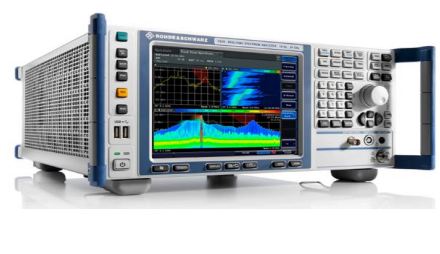

(b)

Figura 2. (a) EMSAT da ANATEL - Rio de Janeiro. (b) Analisador de Espectro FSVR da R\&S - Especificação em www.rohde-schwarz.com.

Características de RF dos Sinais Recebidos pela EMSAT. Foram coletadas doze portadoras (Tabela 1) de cinco diferentes estações de uma rede em banda C - faixa RF de 3,625

Tabela 1. Características de radiofrequência dos sinais coletados.

\begin{tabular}{|c|c|c|c|c|c|c|c|c|c|}
\hline \# & REMOTA & $\begin{array}{l}\text { PORTADORA } \\
\text { / SINAL }\end{array}$ & UF & $\begin{array}{c}\text { FREQ. DESCIDA } \\
\text { DO SATÉLITE (Hz) }\end{array}$ & $\begin{array}{c}\text { FREQUÊNCIA } \\
\text { EM BANDA L }(\mathbf{H z})\end{array}$ & POLARIZAÇÃo & BW (Hz) & MODULAÇÃO & FEC \\
\hline 1 & R1C1PA & 1 & $\mathbf{P A}$ & 3687562500 & 1462437500 & VERTICAL & 1185000 & 8PSK & $3 / 4$ \\
\hline 2 & R1C2PA & 2 & PA & 3693487500 & 1456512500 & VERTICAL & 1185000 & 8PSK & $3 / 4$ \\
\hline 3 & R1C3PA & 3 & PA & 3682822500 & 1467177500 & VERTICAL & 1185000 & 8PSK & $3 / 4$ \\
\hline 4 & R1C1AC & 1 & $\mathbf{A C}$ & 3912642500 & 1237357500 & VERTICAL & 1185000 & 8PSK & $3 / 4$ \\
\hline 5 & R1C2AC & 2 & $\mathbf{A C}$ & 3914842500 & 1235157500 & VERTICAL & 1185000 & 8PSK & $3 / 4$ \\
\hline 6 & R2C1PA & 1 & $\mathbf{P A}$ & 3716222500 & 1433777500 & VERTICAL & 1185000 & 8PSK & $3 / 4$ \\
\hline 7 & R2C2PA & 2 & $\mathbf{P A}$ & 3713852500 & 1436147500 & VERTICAL & 1185000 & 8PSK & $3 / 4$ \\
\hline 8 & R2C1AC & 1 & $\mathbf{A C}$ & 3910442500 & 1239557500 & VERTICAL & 1185000 & 8PSK & $3 / 4$ \\
\hline 9 & R2C2AC & 2 & $\mathbf{A C}$ & 3908072500 & 1241927500 & VERTICAL & 1185000 & 8PSK & $3 / 4$ \\
\hline 10 & R1C1AM & 1 & AM & 4068102500 & 1081897500 & VERTICAL & 1185000 & 8PSK & $3 / 4$ \\
\hline 11 & R1C2AM & 2 & $\mathbf{A M}$ & 4070472500 & 1079527500 & VERTICAL & 1185000 & 8PSK & $3 / 4$ \\
\hline 12 & R1C3AM & 3 & $\mathbf{A M}$ & 4065732500 & 1084267500 & VERTICAL & 1185000 & 8PSK & $3 / 4$ \\
\hline
\end{tabular}


$\mathrm{GHz}$ a 4,2 GHz. O satélite está na órbita geoestacionária. Para possibilitar a leitura e a coleta de dados, o sinal de RF recebido é convertido para a faixa de banda $\mathrm{L}$ (faixa 950 MHz a 1,525GHz) e segue pela cadeia de RF da EMSAT até o analisador de espectro, onde é realizada a coleta e armazenamento dos dados.

Todos os sinais são recebidos na banda $C$, possuem polarização vertical, e são doze portadoras com 1,185 MHz de largura de banda cada uma. A modulação é 8PSK e o FEC (Forward-Error-Correction) de 3/4. A Figura 3 destaca a imagem no espectro de um dos doze sinais que passou pela coleta de dados.

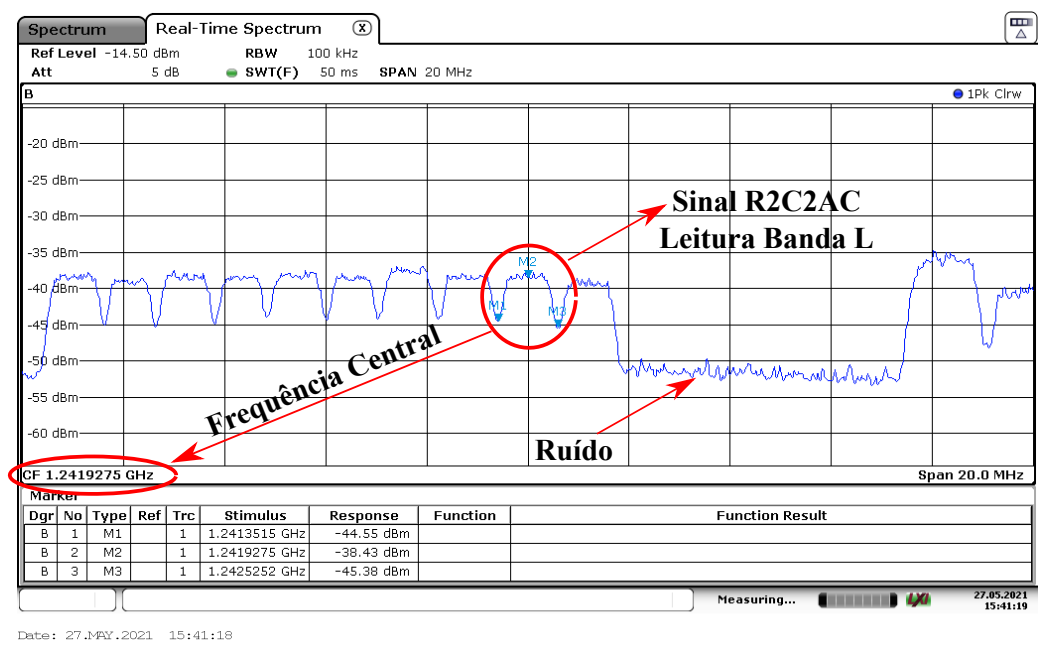

Figura 3. Exemplo de portadora que passou pela coleta de dados.

O Espectrograma. Espectrogramas consistem em um gráfico de intensidade, geralmente em uma escala logarítmica $(\mathrm{dB})$ da magnitude do sinal obtido pelo uso da Transformada Rápida de Fourier (FFT), que fornece informações de frequência localizadas no tempo [Tarongi and Camps 2010]. O espectrograma analisa a densidade espectral de energia, e os valores são apresentados num gráfico de duas dimensões e representados no plano de Tempo vs. Magnitude do sinal em uma dada frequência.

Cada janela do espectrograma cobre 801 dimensões (intervalos de tempo) com uma frequência associada calculada automaticamente pelo analisador de espectro e pela FFT. A Figura 4 (a) mostra a imagem de todo o espectrograma (801 dimensões) para a portadora R2C2AC, enquanto a Figura 4 (b) representa o sinal R2C2AC. E a Figura 4 (c) exemplifica a coleta de sete dimensões (pontos: M1, M2,.., M7) - frequência (Hz) $\mathrm{x}$ amplitude $(\mathrm{dBm})$. Essa representação (Figura 4 (a)) é conhecida como waterfall da portadora onde cada linha/instância contem uma amostra no tempo do sinal coletado.

\section{Coleta e Tratamento do Conjunto SAT-ESPEC}

Fluxo de Coleta. A Figura 5 ilustra o fluxo da transmissão do sinal da remota satélite até a construção da base com os dados brutos. Cada portadora foi armazenada como uma série de instâncias (janelas de sinal coletadas ao longo do tempo ${ }^{2}$ ).

\footnotetext{
${ }^{2} \mathrm{O}$ conjunto SAT-ESPEC está disponível em: https://drive.google.com/drive/ folders/1Feo7jfQnyxpueaC6Sgawzc0 fyCnsv2 JC?usp=sharing
} 
(a)

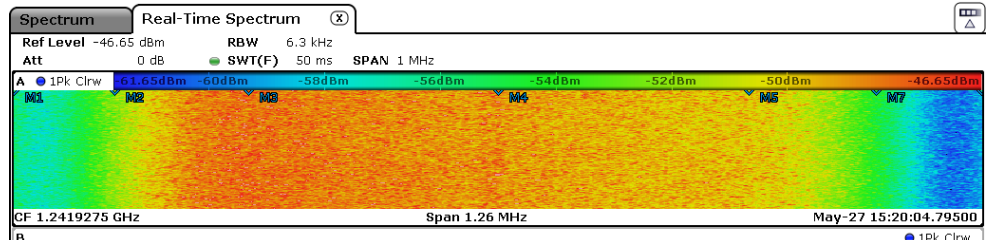

(b)

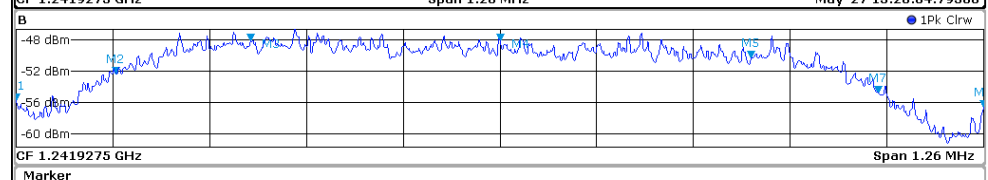

(c)

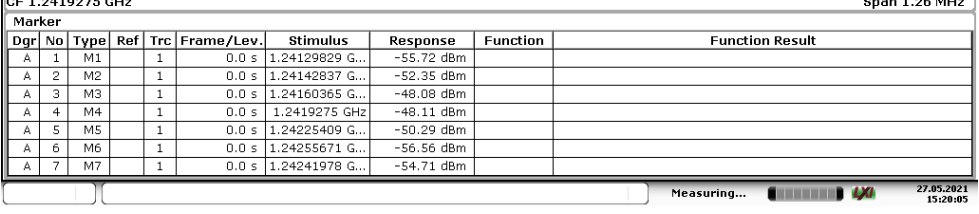

Figura 4. (a) Waterfall de um espectrograma. (b) Representação colunar. (c) tabela com sete dimensões coletadas.

Descrição dos Dados. Os dados brutos armazenados do analisador de espectro apresentam os seguintes elementos:

- Frame. Representa uma coleta do sinal como dado bruto. Cada frame possui um identificador que vai de -299 a 0 , onde o primeiro valor (-299) representa a primeira coleta e assim por diante. Os frames são armazenados em ordem decrescente de tempo na base bruta, sendo que cada frame foi coletado em um intervalo de 50ms. Cada frame foi coletado 300 vezes ao longo do tempo, o que implica a existência de 300 frames com o mesmo identificador e diferentes timestamps. Cada frame pode ser visto como uma instância de um objeto para o instante de tempo definido por sua janela de timestamp.

- Matriz A. Após o frame, a base bruta apresenta uma matriz com 801 linhas por 2 colunas, que foi denominada de "Matriz A": $A_{801 \times 2}$. Nessa matriz, a primeira coluna traz o valor da frequência em $\mathrm{Hz}$, e a segunda coluna traz o valor do nível/amplitude em dBm. A entrada exemplo na Tabela 2 traz uma amostra de um Frame e sua Matriz A associada.

O conjunto SAT-ESPEC em seu formato bruto inclui a Matriz A para cada um dos 300 Frames coletados 300 vezes ao longo do tempo para cada uma das 12 portadoras.

Tabela 2. Exemplo da base bruta coletada no analisador de espectro.

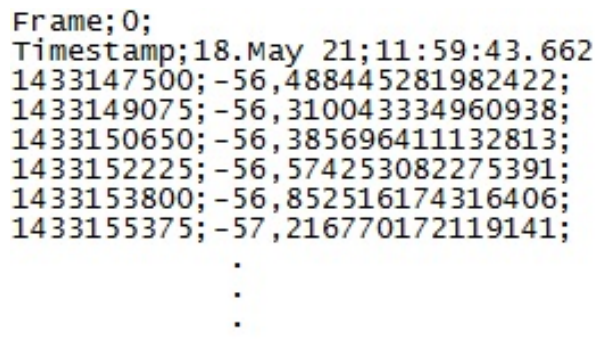

Tratamento Inicial da Base. O processo de tratamento da base bruta para construção do conjunto SAT-ESPEC ocorreu em duas etapas. Primeiro, foi utilizado um script em 


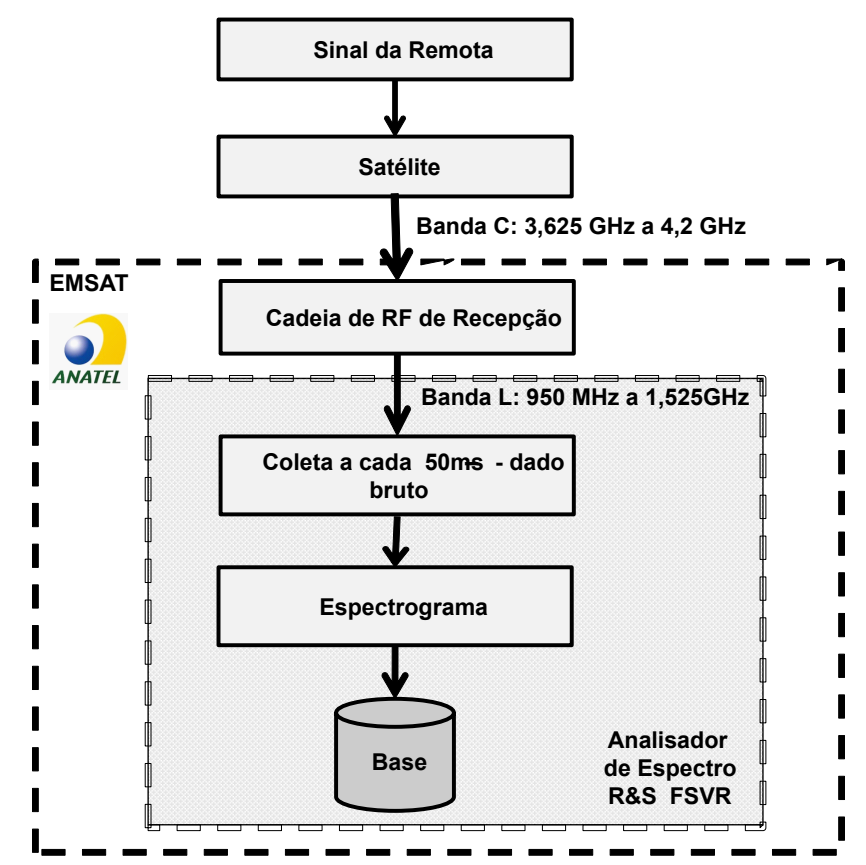

Figura 5. Fluxo da coleta dos dados dos sinais transmitidos pela remota satélite.

Python - contrução Matriz B. Depois, foi realizada uma segunda etapa em um Editor de Planilhas - contrução Matriz C.

Matriz B. A Matriz B propôs uma alteração na estrutura dos dados brutos, onde o mesmo seria reagrupado para um formato de uma matriz com 240300 linhas e 5 colunas. A quantidade de linhas vem do produto do número de frames (300) pela quantidade de coletas (801) realizadas em cada sinal. Para realizar esta alteração, foi utilizado um script desenvolvido em Python. A Figura 6 mostra o dataset após o tratamento da base bruta.

Matriz C. Após a eliminação dos cabeçalhos da base bruta, foi realizada a conversão dos Frames em instâncias identificadas por número e timestamp e sobre eles foi realizada uma amostragem temporal (com intervalos de tempo uniformemente espaçados) para reduzir o tamanho do conjunto de dados base a ser disponibilizado. Como resultado final, foi obtido um conjunto de 3.600 instâncias por 1.605 dimensões. As três primeiras dimensões são: "Identificador do Frame", "Data" e "Hora", enquanto as demais colunas apresentam o valor da frequência e nível do sinal de forma intercalada para 801 níveis. A Figura 7 ilustra a modificação da Matriz B para a Matriz C.

Limpeza e Normalização. Por último, mas não menos importante, a Matriz C foi limpa com o objetivo de se eliminar dados redundantes e reduzir o número de dimensões. Os valores das frequências foram removidos por serem comuns a todas as instâncias, bem como foram suprimidos os identificadores do Frame e Timestamps. O identificador da portadora foi adicionado como meta-atributo. Serão 12 atributos de classe, e cada um representa uma das portadoras coletadas do satélite - atributo "carrier". Esse conjunto de dados limpo e escalado foi exportado para os formatos colunares (.csv) e AttributeRelation (.arff). 


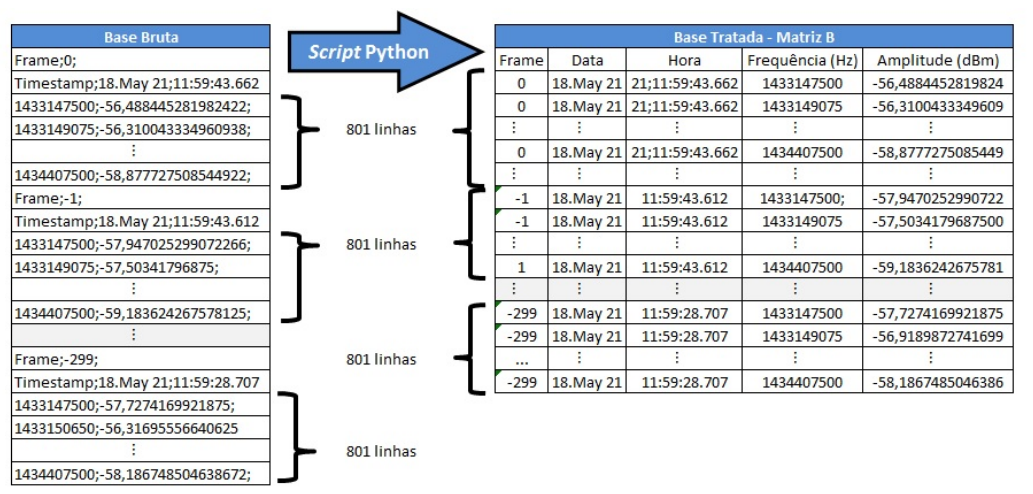

Figura 6. Tratamento da base bruta e construção Matriz B.

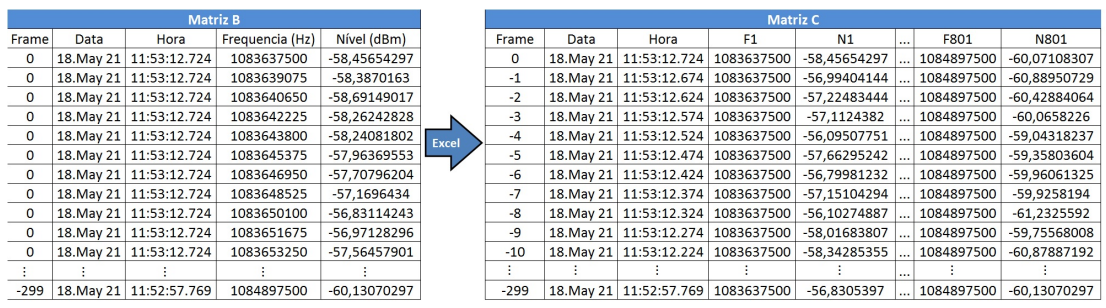

Figura 7. Transformação da Matriz B para a Matriz C.

\section{Sugestões para Utilização do Conjunto de Dados SAT-ESPEC e Considerações Finais}

Os dados coletados das portadoras dos satélites permitem aplicações relacionadas ao estudo de propagação do sinal, segurança de rede, defesa e comportamento em geral de uma rede que utiliza satélite Geoestacionário. Em particular, o conjunto de dados pode ser usado como entrada para ferramentas de aprendizado de máquina em tarefas que envolvam classificação, detecção de anormalidades e análise de séries-temporais.

A extração de características radioelétricas de um sinal e a utilização de algoritmos de aprendizado de máquina para fins de classificação, mostraram-se promissores para a identificação de sinais no âmbito de redes atendidas por satélite. Isto pode ser realizado apenas com a análise da camada física (onda eletromagnética), o que permite criar assinaturas para o que é transmitido, sem a necessidade de protocolos elaborados. $\mathrm{O}$ deslocamento de uma portadora no espectro, sem o devido aviso ou coordenação, também pode ser rastreado com uma abordagem baseada em classificação.

Para ilustrar essa possibilidade de uso do conjunto de dados, são apresentados dois resultados derivados de treinamento de modelos de aprendizado para tarefas de classificação, cujo objetivo é identificar a portadora do satélite dadas as frequências coletadas pelo analisador de espectro. Nesse sentido, os dados do SAT-ESPEC são usados como características supervisionadas que poderiam treinar modelos capazes de identificar sinais de uma portadora, o que aumentaria a proteção contra riscos de invasão (do espectro do sinal de satélite) e acesso não autorizado.

Para essa análise de exemplo, foi utilizado o framework $\mathrm{Weka}^{3}$ e dois diferen-

\footnotetext{
${ }^{3}$ Disponível em cs.waikato.ac.nz/ml/weka/
} 
tes classificadores, avaliados com o método de validação cruzada seguindo a abordagem 10-folds cross-validation. Como parâmetros de treinamento dos classificadores, foram utilizados os seguintes valores:

- Árvore de Decisão (Decision-Tree - C4.5) - Com poda, entropia e split binário.

- Multilayer Perceptron (MLP) - Uma rede shallow, com apenas uma camada oculta de $\lceil\sqrt{801}\rceil$ neurônios.

A Figura 8 apresenta os resultados para esses diferentes classificadores sobre o conjunto SAT-ESPEC com relação a matriz de confusão sobre as portadoras. Como pode ser observado, os classificadores tiveram um razoável desempenho da identificação das portadoras com erros pontuais que representam portadoras dentro do mesmo estado do país e/ou codificadas com frequências muito próximas. Um impacto direto desse resultado é poder identificar qual portadora pode estar efetuando uma transmissão e, com isso, designar rapidamente um especialista de telecomunicações para averiguar e investigar (in loco) o que pode estar ocorrendo no caso de uma interferência ou uso inadequado do canal. Esse procedimento, se realizado na forma de tentativa-e-erro (sem o apoio de um método de classificação ou análise) é extremamente dispendioso em termos de equipamento e pessoal, dadas as dimensões continentais do Brasil.

\begin{tabular}{|c|c|c|c|c|c|c|c|c|c|c|c|c|c|}
\hline & \multicolumn{12}{|c|}{ Árvore de Decisão (C4.5) } \\
\hline & & \multicolumn{12}{|c|}{ Classificado como: } \\
\hline & & 1 & 2 & 3 & 4 & 5 & 6 & 7 & 8 & 9 & 10 & 11 & 12 \\
\hline R1C1PA & 1 & 228 & 12 & 2 & 0 & 0 & $\overline{0}$ & $\overline{0}$ & 0 & 3 & 1 & $\overline{0}$ & 54 \\
\hline \begin{tabular}{|l|} 
R1C2PA \\
\end{tabular} & 2 & 25 & 260 & 3 & 0 & 0 & 12 & $\mathbf{0}$ & $\mathbf{0}$ & 0 & 0 & $\mathbf{0}$ & 0 \\
\hline R1C3PA & 3 & 7 & 6 & 286 & 0 & 0 & 0 & $\mathbf{0}$ & 0 & 0 & 0 & $\mathbf{0}$ & 1 \\
\hline R1C1AC & 4 & 0 & 0 & 0 & 173 & 90 & $\mathbf{0}$ & $\mathbf{0}$ & 28 & 9 & o & 0 & 0 \\
\hline R1C2AC & 5 & 0 & 0 & 0 & 83 & 138 & 0 & $\mathbf{0}$ & 61 & 18 & 0 & $\mathbf{0}$ & $\mathbf{0}$ \\
\hline R2C1PA & 6 & 1 & 8 & $\mathbf{0}$ & 0 & $\mathbf{0}$ & 291 & $\mathbf{0}$ & 0 & 0 & $\mathbf{0}$ & 0 & $\mathbf{0}$ \\
\hline $\mathrm{R2C2PA}$ & 7 & 0 & 1 & 0 & 0 & 0 & 0 & 297 & 0 & 0 & 0 & 2 & $\mathbf{0}$ \\
\hline R2C1AC & 8 & 0 & $\mathbf{0}$ & 1 & 11 & 74 & $\mathbf{0}$ & $\mathbf{0}$ & 191 & 23 & $\mathbf{0}$ & 0 & 0 \\
\hline $\mathrm{R} 2 \mathrm{C} 2 \mathrm{AC}$ & 9 & 5 & $\mathbf{0}$ & 0 & 2 & 2 & 0 & $\mathbf{0}$ & 17 & 229 & 0 & $\mathbf{0}$ & 45 \\
\hline R1C1AM & 10 & 0 & $\mathbf{0}$ & $\mathbf{0}$ & 0 & 0 & $\mathbf{0}$ & $\mathbf{0}$ & 0 & 1 & 299 & 0 & 0 \\
\hline R1C2AM & 11 & 0 & 0 & 0 & 0 & $\mathbf{0}$ & $\mathbf{0}$ & $\mathbf{0}$ & 0 & 0 & $\mathbf{0}$ & 300 & 0 \\
\hline \begin{tabular}{|l|} 
R1C3AM \\
\end{tabular} & 12 & 48 & 1 & 3 & 0 & $\mathbf{0}$ & $\mathbf{0}$ & 0 & 1 & 49 & 0 & 0 & 198 \\
\hline
\end{tabular}

\begin{tabular}{|c|c|c|c|c|c|c|c|c|c|c|c|c|c|}
\hline & \multicolumn{12}{|c|}{ Multilayer Perceptron (MLP) } \\
\hline & & \multicolumn{12}{|c|}{ Classificado como: } \\
\hline & & 1 & 2 & 3 & 4 & 5 & 6 & 7 & 8 & 9 & 10 & 11 & 12 \\
\hline R1C1PA & 1 & 270 & 0 & 0 & 0 & 0 & $\overline{0}$ & $\overline{0}$ & $\overline{0}$ & 1 & 9 & 10 & 10 \\
\hline R1C2PA & 2 & 81 & 150 & 38 & $\mathbf{0}$ & 0 & $\mathbf{0}$ & $\mathbf{0}$ & $\mathbf{0}$ & 0 & 28 & 3 & 0 \\
\hline R1C3PA & 3 & 52 & 0 & 210 & $\mathbf{0}$ & 0 & 0 & $\mathbf{0}$ & $\mathbf{0}$ & 0 & 38 & 0 & $\mathbf{0}$ \\
\hline R1C1AC & 4 & 0 & $\mathbf{0}$ & $\mathbf{0}$ & 286 & 14 & $\mathbf{0}$ & 0 & 0 & 0 & 0 & 0 & 0 \\
\hline R1C2AC & 5 & 0 & 0 & 0 & 14 & 285 & 0 & 0 & 1 & 0 & 0 & 0 & 0 \\
\hline R2C1PA & 6 & 1 & $\mathbf{0}$ & $\mathbf{0}$ & 0 & 0 & 150 & 0 & 0 & 0 & $\mathbf{0}$ & 132 & 17 \\
\hline R2C2PA & 7 & 29 & $\mathbf{0}$ & $\mathbf{0}$ & 0 & 0 & 5 & 120 & 0 & 12 & 0 & 134 & 0 \\
\hline \begin{tabular}{|l|} 
R2C1AC \\
\end{tabular} & 8 & 0 & $\mathbf{0}$ & $\mathbf{0}$ & 1 & 2 & 0 & 0 & 297 & 0 & $\mathbf{0}$ & 0 & 0 \\
\hline R2C2AC & 9 & 0 & $\mathbf{0}$ & $\mathbf{0}$ & 0 & 0 & $\mathbf{0}$ & 0 & 1 & 299 & $\mathbf{0}$ & $\mathbf{0}$ & 0 \\
\hline R1C1AM & 10 & 0 & $\mathbf{0}$ & $\mathbf{0}$ & 0 & 0 & 0 & 0 & 0 & $\mathbf{0}$ & 300 & 0 & 0 \\
\hline R1C2AM & 11 & 0 & 0 & $\mathbf{0}$ & 0 & 0 & 0 & 0 & 0 & $\mathbf{0}$ & $\mathbf{0}$ & 300 & 0 \\
\hline \begin{tabular}{|l|} 
R1C3AM \\
\end{tabular} & 12 & 0 & 0 & 0 & 0 & 0 & 0 & 0 & 0 & 0 & 0 & 0 & 300 \\
\hline
\end{tabular}

( b )

Legenda Melhor Acerto

Figura 8. Matrizes de confusão para diferentes classificadores sobre o conjunto de dados SAT-ESPEC . (a) C4.5 e (b) MLP. 
Agradecimentos. Os autores agradecem a Fundação de Amparo à Pesquisa do Estado do Rio de Janeiro (FAPERJ), a Fundação de Amparo à Pesquisa do Estado de São Paulo (FAPESP), Processo N. 2021/06564-0, CNPq e CAPES pelo apoio financeiro para a realização desse estudo. Josinaldo Azevedo encontra-se em afastamento pela ANATEL junto ao Instituto Militar de Engenharia. Marcos Bedo encontra-se em afastamento pela Universidade Federal Fluminense junto ao HC-FMRP/USP.

\section{Referências}

Bitar, N., Muhammad, S., and Refai, H. H. (2017). Wireless technology identification using deep convolutional neural networks. In 2017 IEEE 28th Annual International Symposium on Personal, Indoor, and Mobile Radio Communications, pages 1-6.

Özseven, T. (2018). Investigation of the effect of spectrogram images and different texture analysis methods on speech emotion recognition. Applied Acoustics, 142:70-77.

Pratt, T. and Allnutt, J. (2020). Satellite Communication. 2020 John Wiley \& Sons Ltd, Virginia, USA, 3 edition.

Tarongi, J. and Camps, A. (2010). Radio frequency interference detection algorithm based on spectrogram analysis. In 2010 IEEE International Geoscience and Remote Sensing Symposium, pages 2499-2502.

Wang, W., Zhang, G., Yang, L., Balaji, V., Elamaran, V., and Arunkumar, N. (2019). Revisiting signal processing with spectrogram analysis on eeg, ecg and speech signals. Future Generation Computer Systems, 98:227-232. 